\title{
Herbal pair Huangqin-Baishao: mechanisms underlying inflammatory bowel disease by combined system pharmacology and cell experiment approach
}

Xiaoqi Huang ${ }^{1,2+}$, Zhiwei Chen ${ }^{2+}$, Minyao Li $i^{1}$, Yaomin Zhang ${ }^{1,2}$, Shijie X ${ }^{1}$, Haiyang Huang ${ }^{2}$, Xiaoli $\mathrm{Wu}^{3^{*}}$ and Xuebao Zheng ${ }^{1,4^{*}}$

\begin{abstract}
Background: Inflammatory bowel disease (IBD) is a severe digestive system condition, characterized by chronic and relapsing inflammation of the gastrointestinal tract. Scutellaria baicalensis Georgi (Huangqin, $\mathrm{HQ}$ ) and Paeonia lactiflora Pall (Baishao, BS) from a typical herbal synergic pair in traditional Chinese medicine (TCM) for IBD treatments. However, the mechanisms of action for the synergy are still unclear. Therefore, this paper aimed to predict the anti-IBD targets and the main active ingredients of the HQ-BS herbal pair.

Methods: A systems pharmacology approach was used to identify the bioactive compounds and to delineate the molecular targets and potential pathways of HQ-BS herbal pair. Then, the characteristics of the candidates were analyzed according to their oral bioavailability and drug-likeness indices. Finally, gene enrichment analysis with DAVID Bioinformatics Resources was performed to identify the potential pathways associated with the candidate targets.

\footnotetext{
* Correspondence: wuxiaoli@gdut.edu.cn; xuebaozheng@gzucm.edu.cn ${ }^{+}$Xiaoqi Huang and Zhiwei Chen contributed equally to this work.

${ }^{3}$ School of Biomedical and Pharmaceutical Sciences, Guangdong University of Technology, 100\# Wai Huan West Road, Guangzhou Higher Education Mega Center, Guangzhou 510006, China

${ }^{1}$ Mathematical Engineering Academy of Chinese Medicine, Guangdong Provincial Key Laboratory of New Chinese Medicinal Development and Research, Guangzhou University of Chinese Medicine, 232\# Wai Huan East Road, Guangzhou Higher Education Mega Center, Guangzhou 510006, China Full list of author information is available at the end of the article
}

(c) The Author(s). 2020 Open Access This article is licensed under a Creative Commons Attribution 4.0 International License, which permits use, sharing, adaptation, distribution and reproduction in any medium or format, as long as you give appropriate credit to the original author(s) and the source, provide a link to the Creative Commons licence, and indicate if changes were made. The images or other third party material in this article are included in the article's Creative Commons. licence, unless indicated otherwise in a credit line to the material. If material is not included in the article's Creative Commons licence and your intended use is not permitted by statutory regulation or exceeds the permitted use, you will need to obtain permission directly from the copyright holder. To view a copy of this licence, visit http://creativecommons.org/licenses/by/4.0/ The Creative Commons Public Domain Dedication waiver (http://creativecommons.org/publicdomain/zero/1.0/) applies to the data made available in this article, unless otherwise stated in a credit line to the data. 
(Continued from previous page)

Results: The results showed that, a total of 38 active compounds were obtained from HQ-BS herbal pair, and 54 targets associated with IBD were identified. Gene Ontology and pathway enrichment analysis yielded the top 20 significant results with 54 targets. Furthermore, the integrated IBD pathway revealed that the HQ-BS herbal pair probably acted in patients with IBD through multiple mechanisms of regulation of the nitric oxide biosynthetic process and anti-inflammatory effects. In addition, cell experiments were carried out to verify that the HQ-BS herbal pair and their Q-markers could attenuate the levels of nitric oxide (NO), prostaglandin $E_{2}\left(P_{G} E_{2}\right)$, inducible nitric oxide synthase (iNOS) and cyclooxygenase-2 (COX-2) in lipopolysaccharide (LPS)-stimulated THP-1-derived macrophage inflammation. In particular, the crude materials exerted a much better anti-inflammatory effect than their Q-markers, which might be due to their synergistic effect.

Conclusion: This study provides novel insight into the molecular pathways involved in the mechanisms of the HQ-BS herbal pair acting on IBD.

Keywords: HQ-BS herbal pair, Inflammatory bowel disease, Synergic mechanism, System pharmacology, THP-1 cells

\section{Background}

Inflammatory bowel disease (IBD) including Crohn's disease (CD) and ulcerative colitis (UC), is a chronic and recurring intestinal inflammatory disorder. Although a number of commercial drugs have been used to treat IBD in the clinic, a lack of sustained efficacy and side effects hinder their application. It has been reported that one-third of UC patients and two-thirds of CD patients need to undergo surgery eventually $[1,2]$. TCM has been widely used to treat different kinds of diseases in China for a long time. TCM usually consist of different herbal medicines mixed together in specific quantities to form a formula according to the composition theory "Monarch, Minister, Assistant, and Guide". In the formula, Monarch means the main drug for the etiology and symptoms; Minister means promoting the therapeutic effect produced by Monarch; Assistant means treating the accompanying symptom or eliminating the side effects of the Monarch; and Guide means guiding the formula to the disease area or reconciling its efficacy [3-5]. To improve the quality of life, it is vital to develop nontoxic and sustained efficacy medicines to treat IBD.

Studies have reported various beneficial effects of TCM and herbal extracts in treating IBD including antibacterial, anti-inflammatory, and anti-cancer activities $[6,7]$. Among them, HuangQin decoction (HQD) is a famous formula recorded in the Treatise on Exogenous Febrile Disease written by Zhongjing Zhang. HQD has been used to treat gastrointestinal disease such as diarrhea, abdominal spasms, vomiting, and nausea for a long time in China [8]. HQD consists of four ingredients: the roots of Scutellaria baicalensis Georgi (Huangqin, HQ), Paeonia lactiflora Pall (Baishao, BS), Glycyrrhiza uralensis Fisch (Gancao, GC), and the fruit of Ziziphus jujuba Mill (Dazao, DZ) [9]. HQD has been proven to ameliorate intestinal damage [10]. According to the composition theory, HQ is the Monarch, and BS is the Minister in this formula. HQ-BS is composed of a typical herbal synergic pair and is widely used for colitis treatments. PF2405, a standardized fraction of Scutellaria baicalensis, shows prominent anti-inflammatory action in ameliorating colitis in vitro and in vivo [11]. Moreover, Dai et al. used a system pharmacology method and experiments to discover and prove Q-markers of HQD in the role of attenuating IBD, and nine constituents (paeoniflorin, baicalin, scutellarein, liquiritigenin, norwogonin, baicalein, glycyrrhizic acid, wogonin, and oroxylin A) were found to be Q-markers of HQD. Moreover, all of these compounds are the main constituents of HQ-BS [9]. Baicalin was proved to exert a remarkable anti-inflammatory effect on colitis induced by dextran sodium sulfate (DSS), and the mechanistic investigation revealed that it may be regulated through the caudal-type homeobox 2 (CDX2/ pregnane $\mathrm{X}$ receptor (PXR) pathway $[12,13]$. Furthermore, Luo and colleagues provided evidence that baicalin attenuated colitis induced by trinitro-benzenesulfonic acid (TNBS) via inhibiting the toll like receptor4. (TLR4)/myeloid differential protein-88 (MyD88) signaling cascade and inactivating the NOD-like receptor 3 (NLRP3) inflammasome [14]. Zhang et al. proved that paeoniflorin abrogated colitis induced by DSS via reducing the expression of TLR4 and inhibiting the activation of nuclear factor kappa-B (NF-kB) and the mitogenactivated protein kinase (MAPK) pathway [15]. Gu et al. showed that paeoniflorin exerted anti-inflammatory and anti-apoptosis actions in ulcerative colitis by suppressing the MAPK/NF- $\mathrm{B}$ pathway in mice [16].

Although HQD and its bioactive components exert significant effects in attenuating IBD, the specific bioactive molecules in HQD and the underlying mechanisms of action remain unknown. Currently, system pharmacology has successfully been widely used to investigate the role and the mechanism of TCM [17-23]. Thus, in the current study, we use a system pharmacology approach combined with cell experiments (the protocol is shown in Fig. 1) to investigate the active 


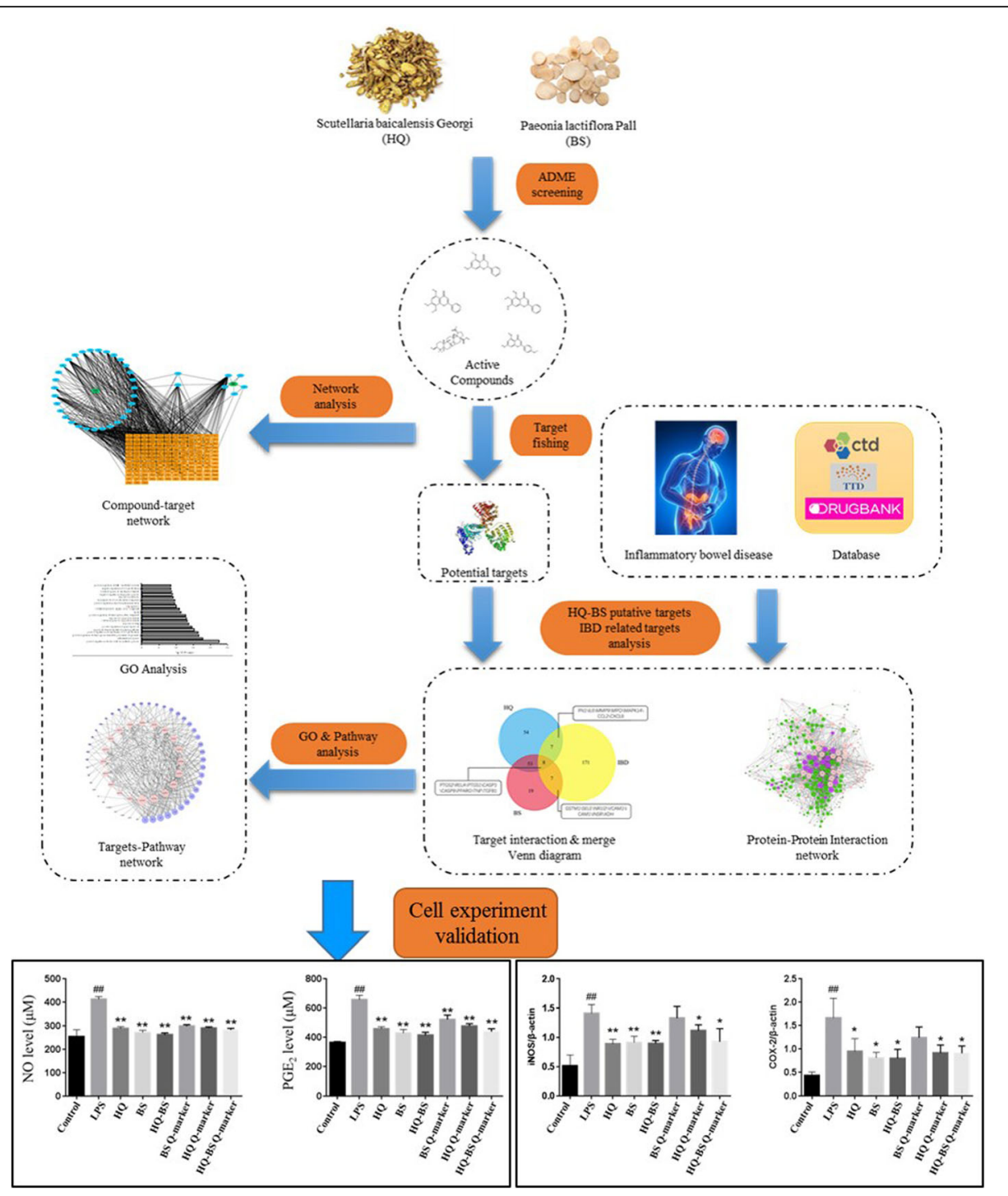

Fig. 1 Systems pharmacology approach workflow for elucidating the mechanisms of actions for the synergy of the HQ-BS herbal pair IBD

constituents, targets and underlying pathways of the HQ-BS herbal pair.

\section{Materials and methods}

\section{Data preparation}

The chemical compound data and the targets of HQ and BS were obtained from the Traditional Chinese Medicine Systems Pharmacology Database (TCMSP, http:// sm.nwsuaf.edu.cn/lsp/tcmsp.php.) [24] and were manually supplemented. Target information of the ingredients was obtained from DrugBank (www.drugbank.ca) [25]. Anti-IBD targets were derived from the Therapeutic Targets Database (TTD, http://bidd.nus.edu.sg/group/ ttd/ttd.asp) [26], Comparative Toxicogenomics Database (CTD, http://ctdbase.org/) [27] and PharmGKB (http:// www.pharmgkb.org) [28]. Protein-protein interaction
(PPI) data were derived from the STRING database (http://string-db.org) [29]. Putative targets were enriched in the Kyoto Encyclopedia of Genes and Genomes (KEGG, http://www.kegg.jp/ or http://www.genome.jp/ $\mathrm{kegg} /$ ) [30]. All of the targets were mapped to the database UniProt (http://www.uniprot.org) [31] to construct the networks of Herb-Compound-Target (H-C-T).

\section{Network construction and network analysis}

Networks were constructed using the data about the active constituents, corresponding targets, and pathway information. Moreover, we used an open software package project named Cytoscape v1.1 Core (http://www.cytoscape.org/) [32] to construct all of the visualized network graphs. 


\section{Gene ontology and pathway enrichment analysis}

Gene Ontology (GO) enrichment analysis for the putative targets of the HQ and BS herbal pair was conducted using the Database for Annotation, Visualization and Integrated Discovery (DAVID, https://david.ncifcrf. gov) [33]. DAVID was also applied to perform pathway enrichment analysis for the same gene targets to identify the potential synergistic actions of $\mathrm{HQ}$ and BS against IBD. Enriched GO terms and pathways were defined as those with false discovery rate (FDR) adjusted $P$ values $<0.05$.

\section{Cell culture}

THP-1 cells (human monocytes derived from a patient with acute monocytic leukemia) were cultured with RPMI 1640 medium containing 10\% fetal bovine serum and $1 \%$ penicillin-streptomycin at $37^{\circ} \mathrm{C}$ under a humidified atmosphere with $5 \%(v / v) \mathrm{CO}_{2}$.

\section{Chemicals and reagents}

LPS (L4391, a purity no less than 99\%) was purchased from Sigma (St. Louis, USA,); HQ and BS crude materials were obtained from Kangmei Pharmaceutical Co. LTD (Jieyang, China) and authenticated by Prof. Ziren $\mathrm{Su}$ (Guangzhou University of Chinese Medicine); paeoniflorin (CAS: 23180-57-6), baicalin (CAS: 21967-41-9), baicalein (CAS: 352000-07-8), wogonin (CAS: 632-859), and oroxylin A (CAS: 23180-57-6) were purchased from Dalian Meilun Biotechnology Co., Ltd. (Dalian, China), the purities are no less than 98\%; NO assay kit (A013-2-1) was purchased from Nanjing Jiancheng Bioengineering Institute (Nanjing China); $\mathrm{PGE}_{2}$ (CSBE07966m), iNOS (CSB-E08326m) and COX-2 (CSBE12910m) ELISA kits were supplied from CUSABIO (Wuhan China). The iNOS anti-body (AF0199) and COX-2 anti-body (AF7003) were purchased from Affinity Biosciences Inc. (Cincinnati, USA).

\section{Drug groups design}

LPS-stimulated THP-1-derived macrophage inflammation was used as a cell model to assess the Q-markers and crude medicines from the HQ-BS herbal pair. We designed the groups as follows: HQ crude material $(51 \mu \mathrm{g} / \mathrm{ml}), B S$ crude material $(34 \mu \mathrm{g} / \mathrm{ml})$, HQ-BS crude material pair $(85 \mu \mathrm{g} / \mathrm{ml})$, BS Q-markers (paeoniflorin $3.218 \mu \mathrm{g} / \mathrm{ml}$ ), HQ Q-markers (baicalin $17.026 \mu \mathrm{g} / \mathrm{ml}+$ baicalein $0.59 \mu \mathrm{g} / \mathrm{ml}+$ wogonin $0.316 \mu \mathrm{g} / \mathrm{ml}+$ oroxylin A $0.1 \mu \mathrm{g} / \mathrm{ml}$ and the HQ-BS herbal pair Q-markers (paeoniflorin $3.218 \mu \mathrm{g} / \mathrm{ml}+$ baicalin $17.026 \mu \mathrm{g} / \mathrm{ml}+$ baicalein $0.59 \mu \mathrm{g} / \mathrm{ml}+$ wogonin $0.316 \mu \mathrm{g} / \mathrm{ml}+$ oroxylin A $0.1 \mu \mathrm{g} /$ $\mathrm{ml})$. The crude materials were extracted with distilled water and concentrated. The Q-markers were dissolved in DMSO and then diluted with culture medium to become the Q-markers of the HQ-BS decoction. The incubation concentration of the materials and the Qmarkers were calculated from $400 \mathrm{mg} / \mathrm{ml}$ of HQD.

\section{$\mathrm{NO}$ and $\mathrm{PGE}_{2}$ release assay}

THP-1 cells were seeded in 96-well plates at 10,000 per well for $12 \mathrm{~h}$. Subsequently, the drugs mentioned above were added to the cells and they were incubated for 12 h. Then, the cells were subjected to $1 \mathrm{mg} / \mathrm{ml}$ LPS for 24 $\mathrm{h}$, except for the vehicle control group. Finally, the culture medium was collected for the $\mathrm{NO}$ and $\mathrm{PGE}_{2}$ assays using commercial ELISA kits according to the manufacturer's instructions.

\section{iNOS and COX-2 assays}

THP-1 cells were seeded in 6-well plates at 100,000 per well for $12 \mathrm{~h}$. Subsequently, the drugs mentioned above were added to the cells and they were incubated for $12 \mathrm{~h}$. Then, the cells were subjected to $1 \mathrm{mg} / \mathrm{ml}$ LPS for $24 \mathrm{~h}$, except for the vehicle control group. Finally, the cells were collected for iNOS and COX-2 assay using Western blot. The cells were lysed in chilled lysis buffer containing protease and phosphatase inhibitors for $20 \mathrm{~min}$ on ice. Afterward, they were centrifuged at $14000 \mathrm{~g}$ and $4{ }^{\circ} \mathrm{C}$ for 10 min and the protein concentration of the supernatant was measured using a BCA Protein Assay Kit according to the protocol provided by the manufacturer. The protein samples were separated on SDS-PAGE gels and then transferred onto PVDF membranes. The membranes were blocked with $5 \%$ skim milk in TBST. Then, the membranes were incubated with iNOS and COX-2 primary antibodies at $4{ }^{\circ} \mathrm{C}$ overnight followed by incubation with the secondary antibodies for $1 \mathrm{~h}$ at room temperature. Finally, the bands were detected by an ECL reagent using a Western blotting detection system.

\section{Statistical analysis}

All data are expressed as the mean \pm standard deviation (SD) and analyzed by one-way analysis of variance (ANOVA) followed by a least-significant difference test. $p$-values of $<0.05$ or $<0.01$ were regarded as statistically significant. The statistical analysis was performed by SPSS 20.0 (SPSS Inc., NY, USA).

\section{Results}

\section{Ingredient comparisons in the HQ-BS herbal pair}

The ingredients in the HQ-BS pair were retrieved from the TCMSP. All of the constituents from the HQ-BS herbal pair were used to construct a compound library. A total of 215 constituents were obtained for HQ (143) and BS (85), respectively. The main ingredients of HQ are flavonoids and alkaloids, and the major constituents in BS are triterpenoids. We evaluated the molecular diversities of all the constituents from the HQ-BS herbal pair based on the following properties as shown in Fig. 2). 


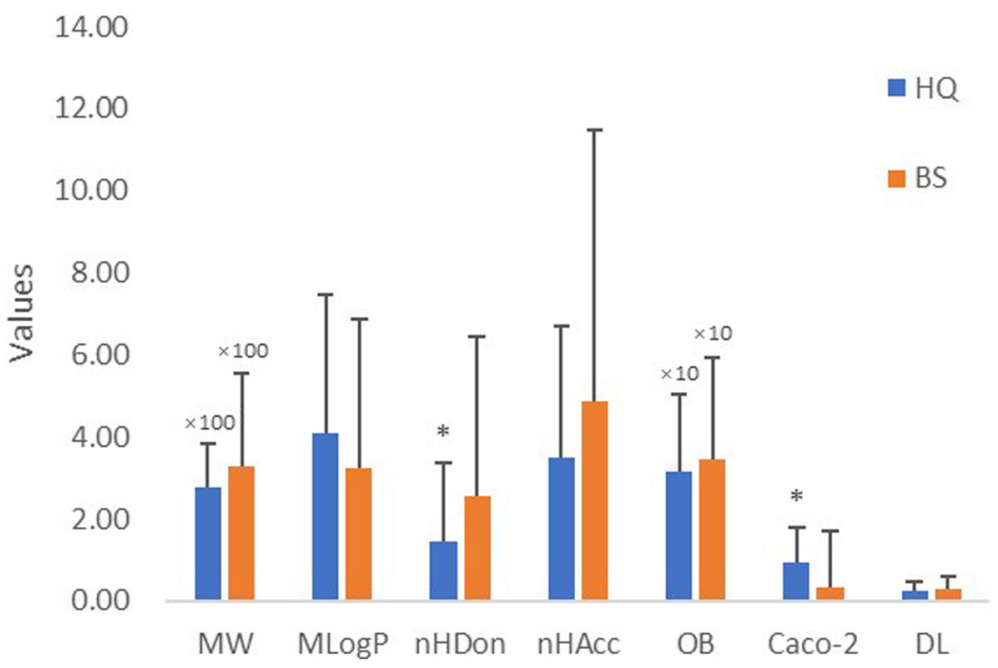

Fig. 2 Properties comparison of ingredients from HQ-BS herbal pair. ${ }^{*} p<0.05$ by two tailed t-test (vs. BS)

(1) The average molecular weight (MW) of each ingredient from HQ and BS was calculated, and there was no significant difference $(p=0.06)$ between HQ (277.74) and BS (326.75).

(2) The Moriguchi octanol-water partition coeff. $(\log \mathrm{P})$ (MLogP) of each ingredient from HQ and BS was calculated, and there was no significant difference $(p=0.07)$ between HQ (4.10) and BS (3.22). This result suggests that hydrotropic constituents are the main ingredients in both $\mathrm{HQ}$ and $\mathrm{BS}$.

(3) The number of donor atoms for the H-bonds (nHDon) of each ingredient from $\mathrm{HQ}$ and BS was calculated, and the average nHDon number of BS constituents (2.55) was much higher than that of the HQ constituents (1.44) $(p=0.02)$.

(4) The number of acceptor atoms for H-bonds (nHAcc) of each ingredient from HQ and BS was calculated and there was no significant difference $(p=0.07)$ between HQ (3.48) and BS (4.86).

(5) The average oral bioavailability (OB) value of the constituents of HQ (31.42) and BS (34.43) was without significant difference $(p=0.33)$.

(6) The Caco-2 permeability Caco-2 of each ingredient from $\mathrm{HQ}$ and $\mathrm{BS}$ was calculated, and the average nHDon number of BS constituents (0.328) was much lower than that of the HQ constituents (0.94) $\left(p=3.58 \times 10^{-4}\right)$.

(7) For drug-likeness (DL) analysis, the average DL index of the constituents of $\mathrm{HQ}$ and BS were 0.23 and 0.29 , respectively, which displays no significant difference $(p=0.11)$.

Despite the constituents of HQ and BS being diverse, based on the analysis above, we also found that the majority of them met the Lipinski's rule of five. Apart from the nHDon, HQ was similar to BS in other properties of the chemical components. These similarities indicate that the constituents of $\mathrm{HQ}$ and $\mathrm{BS}$ share similar chemo-physical properties and that the ingredients from HQ and BS possessed similar DL. These results also indicate that the compounds from the HQ-BS herbal pair had similar pharmacokinetic properties.

\section{Active ingredients in the HQ-BS herbal pair}

Although the HQ-BS herbal pair contains a large number of bioactive compounds, only a few that exert desirable pharmacodynamic and pharmacokinetic properties contribute to the therapeutic actions of this herbal pair. In the current study, we screened the active constituents from the HQ-BS herbal pair from the following absorption, distribution, metabolism, and excretion (ADME) parameters, including OB, Caco-2 and DL. Some constituents were also chosen for their effective bioactivities without satisfying all of these criteria. As a result, a total of 38 active compounds (33 active compounds from HQ and 7 active compounds from BS, and among them, two compounds found in both HQ and BS) were chosen from the 215 compounds of this herbal pair (shown in Supplementary Table 1).

\section{Targets of the HQ-BS herbal pair that are anti-IBD}

To investigate the synergic mechanism of the HQ-BS herbal pair for IBD, the parameters of the active compounds of $\mathrm{HQ}$ and BS were obtained from the TCMSP and the target information was collected from DrugBank. Herb Ingredients' Target (HIT, http://lifecenter. sgst.cn/hit/) [34] was used for specific targets validated by the experiments and the SysDT model [35] was used for the targets without experimental verification. As shown in Supplementary Table 2 and Fig. 3, 147 putative 


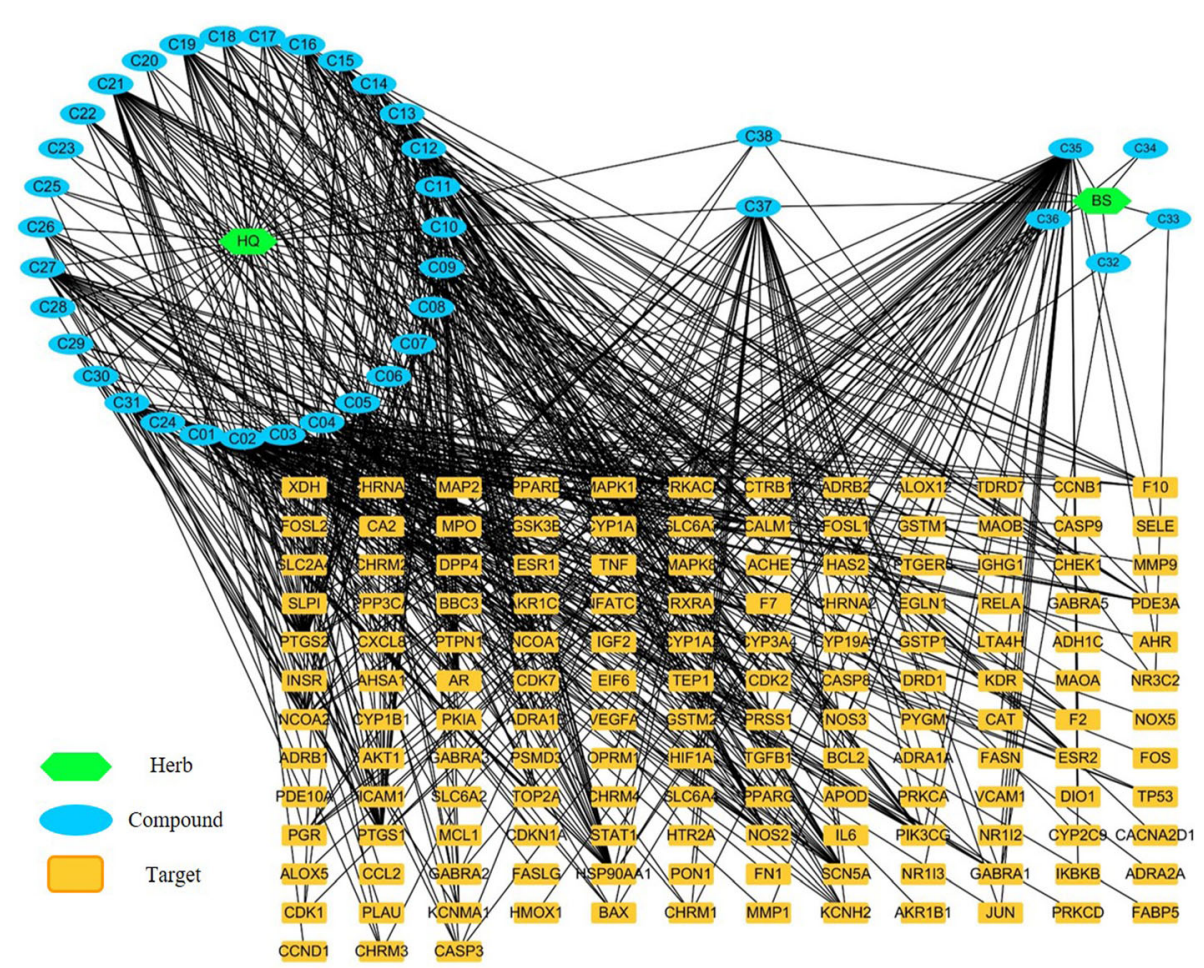

Fig. 3 Herb-Compound-Target (H-C-T) Network. The green nodes represent herbs, the blue nodes represent compounds, and the orange nodes represent $\mathrm{HQ}-\mathrm{BS}$ putative targets, respectively

targets that belong to 38 active ingredients of the HQ-BS pair were collected. All of the above targets were mapped to the database UniProt. Subsequently, we constructed the networks of $\mathrm{H}-\mathrm{C}-\mathrm{T}$ using these data. Finally, we calculated the contribution index of all the active constituents based on the networks.
Interactions between the HQ-BS putative targets and the anti-IBD targets

Targets for IBD were derived from the TTD, CTD and PharmGKB by using the keywords "inflammatory bowel disease (IBD)", "Crohn's disease" and "ulcerative colitis". As shown in Fig. 4, the three target sets, anti-IBD targets (yellow), putative targets for HQ (blue), and putative

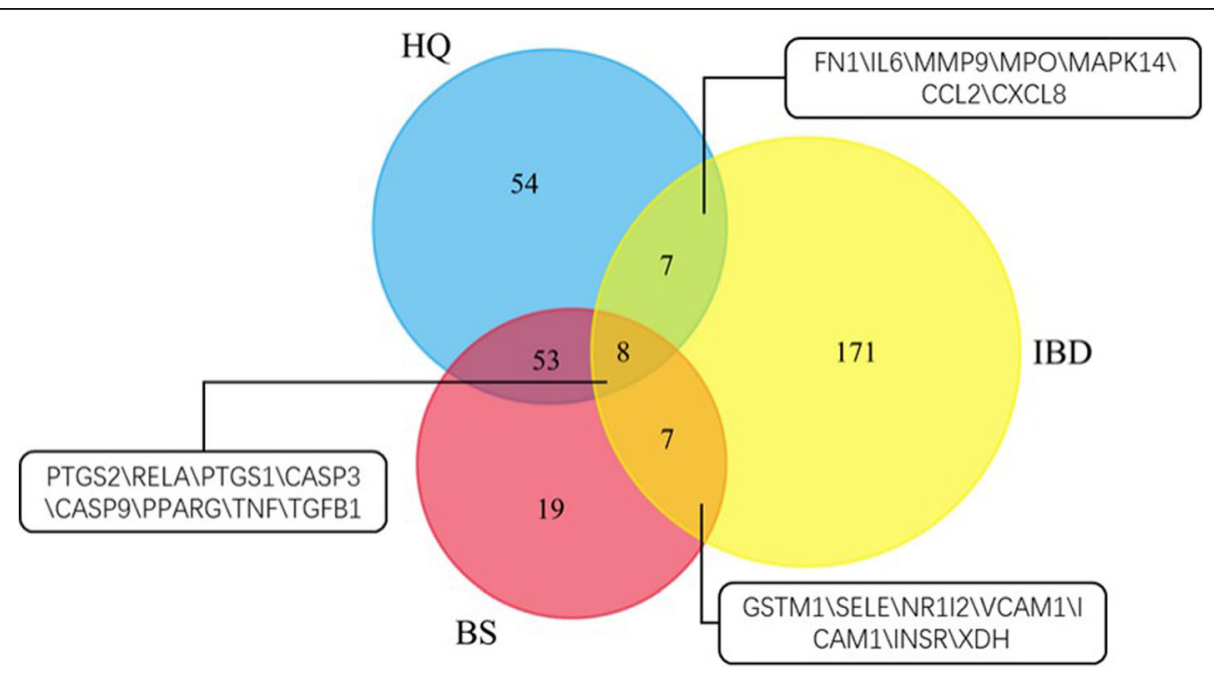

Fig. 4 Direct putative anti-IBD targets of HQ-BS herbal pair. Venn diagram for putative targets of HQ-BS herbal pair and known anti-IBD targets. The targets in each intersection set were also listed 
targets for BS (red), had intersections containing targets. Among the intersected targets, 8 targets were at the intersections of three targets sets, 7 targets were at the intersections of the blue and yellow sets, and 7 targets were at the intersections of the red and yellow sets. The 22 targets were considered to be the direct putative HQBS herbal pair anti-IBD targets.

Next, we constructed a PPI network using the 193 IBD targets and the 147 HQ-BS putative targets. The PPI was ranked among four minimum required interaction scores (low, the score is 0.150; medium, 0.4; high, 0.7; and highest, 0.9). We selected the high scoring (scores > 0.7) PPIs to construct the PPI network. Our analysis obtained a connected network of 319 proteins and 2093 interactions. From them, 66 were anti-IBD targets, 385 were HQ-BS putative targets, and in the intersection lay $26 \mathrm{HQ}-\mathrm{BS}$ against IBD targets. The specific interactions are depicted in Fig. 5a. With a hub node [36] defined as a node with a degree higher than 2-fold of the average degree of all the nodes in the networks (the average degree of all the nodes in the network was 13 in our study), 45 major hub nodes were identified (Fig. 5b).

\section{Functions of the HQ-BS herbal pair for IBD by GO enrichment}

As shown in Table 1 and Fig. 6, GO enrichment analysis of the 54 putative targets of HQ and BS (combining the 22 IBD related targets with 45 important targets) resulted in the top 20 biological processes (BP) with FDR corrected $p<0.05$. The results indicate that they are involved in signal transduction, regulation, and response

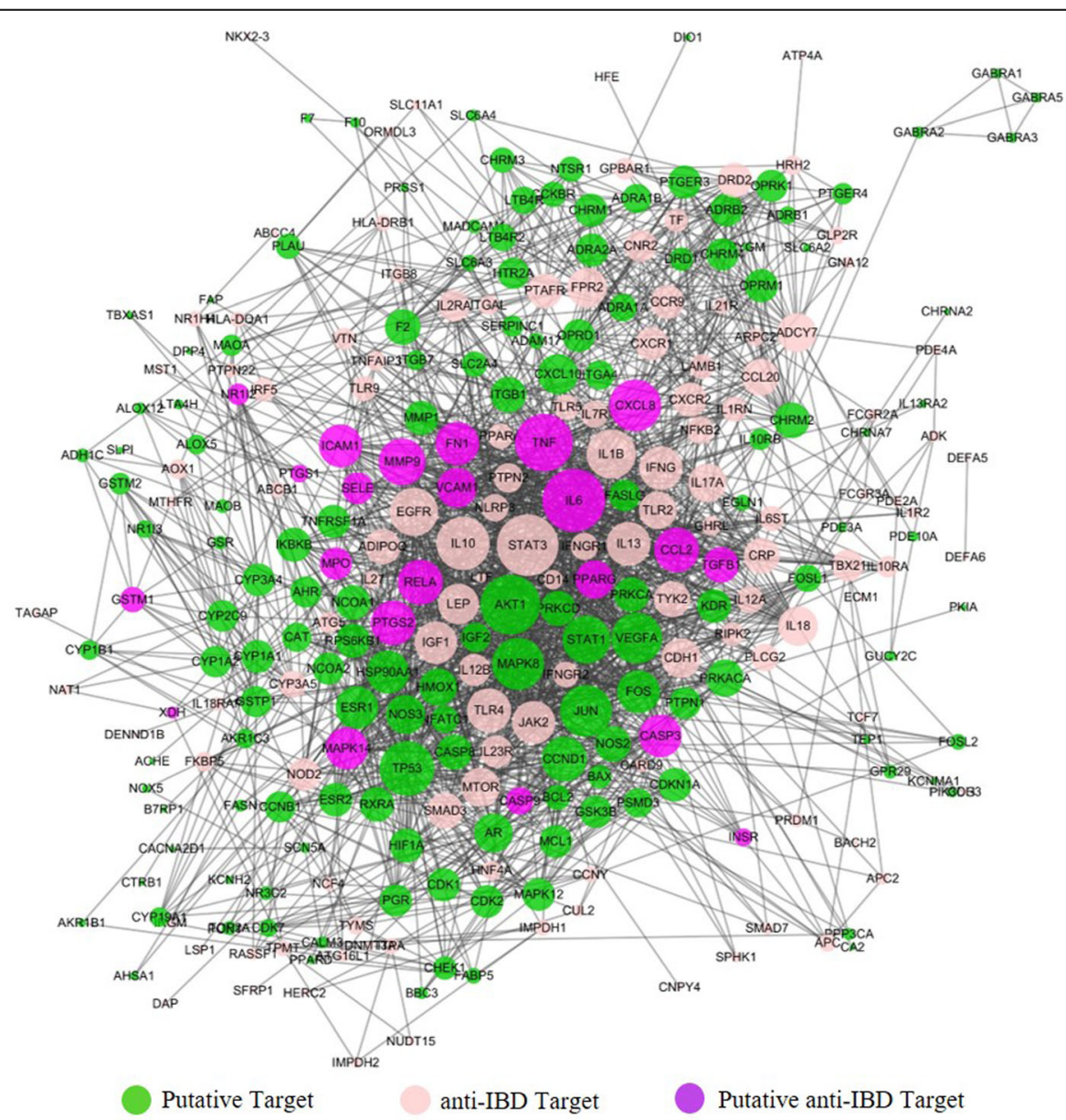

\begin{tabular}{|c|l|}
\hline & VEGFA、MTOR、TP53、CCND1、AKT1、JUN、FOS、CASP3、TNF、NOS3、 \\
45 Important & MAPK8、ICAM1、AR、JAK2、STAT3、IL10、HSP90AA1、PPARG、STAT1 \\
Target & EGFR、IL1B、IGF1、RELA、IL6、IFNG、ESR1、IL18、CXCL8、IL13、CDH1 \\
& CCL2、MAPK14、VCAM1、PRKACA、IL17A、LEP、TLR2、CXCL10、TLR4、 \\
& MMP9、FN1、PTGS2、HMOX1、FPR2、ADCY7 \\
\hline
\end{tabular}

Fig. 5 Protein-protein interaction (PPI) network of anti-IBD targets and proteins that indirectly interact with the targets. The PPI network of antiIBD protein targets (The size of the circle represents the degree of a node); A list of 45 crucial proteins which interact with the anti-IBD targets 
Table 1 The top 20 biological processes enriched by the 54 putative targets

\begin{tabular}{|c|c|c|c|}
\hline Term & Count of Proteins & $\%$ & $p$-Value \\
\hline positive regulation of nitric oxide biosynthetic process & 14 & 25.93 & $2.12 \times 10^{-23}$ \\
\hline inflammatory response & 20 & 37.04 & $1.89 \times 10^{-18}$ \\
\hline positive regulation of transcription from RNA polymerase II promoter & 26 & 48.15 & $1.90 \times 10^{-17}$ \\
\hline positive regulation of smooth muscle cell proliferation & 12 & 22.22 & $3.09 \times 10^{-17}$ \\
\hline lipopolysaccharide-mediated signaling pathway & 10 & 18.52 & $4.01 \times 10^{-16}$ \\
\hline positive regulation of gene expression & 16 & 29.63 & $1.99 \times 10^{-15}$ \\
\hline response to drug & 16 & 29.63 & $1.76 \times 10^{-14}$ \\
\hline cellular response to lipopolysaccharide & 12 & 22.22 & $4.69 \times 10^{-14}$ \\
\hline response to lipopolysaccharide & 13 & 24.07 & $9.47 \times 10^{-14}$ \\
\hline positive regulation of transcription, DNA-templated & 18 & 33.33 & $1.69 \times 10^{-13}$ \\
\hline aging & 12 & 22.22 & $3.15 \times 10^{-12}$ \\
\hline cellular response to organic cyclic compound & 9 & 16.67 & $1.11 \times 10^{-11}$ \\
\hline angiogenesis & 12 & 22.22 & $8.27 \times 10^{-11}$ \\
\hline positive regulation of protein phosphorylation & 10 & 18.52 & $2.04 \times 10^{-10}$ \\
\hline movement of cell or subcellular component & 9 & 16.67 & $2.49 \times 10^{-10}$ \\
\hline response to antibiotic & 7 & 12.96 & $6.28 \times 10^{-10}$ \\
\hline negative regulation of apoptotic process & 14 & 25.93 & $1.12 \times 10^{-9}$ \\
\hline cellular response to mechanical stimulus & 8 & 14.81 & $2.36 \times 10^{-9}$ \\
\hline negative regulation of cell proliferation & 13 & 24.07 & $2.79 \times 10^{-9}$ \\
\hline positive regulation of ERK 1 and ERK2 cascade & 10 & 18.52 & $3.53 \times 10^{-9}$ \\
\hline
\end{tabular}

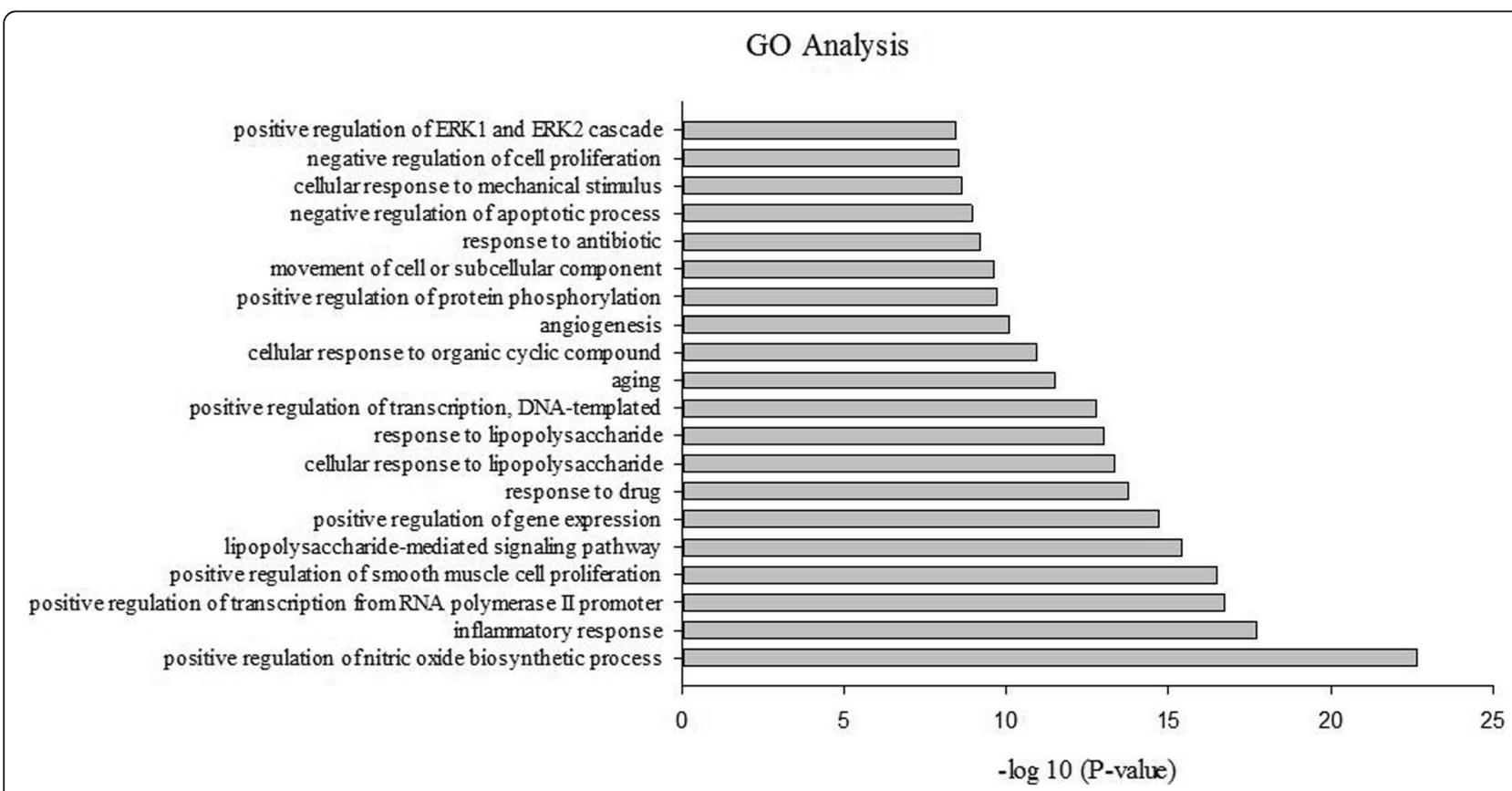

Fig. 6 the top 20 biological processes by GO enrichment analysis of therapeutic targets. The -axis represents the enrichment scores of these terms $(p<0.05)$, and the -axis represents significantly enriched BP categories in GO relative to the targets 
processes, such as positive regulation of nitric oxide biosynthetic process (25.93\%), inflammatory response (37.04\%), lipopolysaccharide-mediated signaling pathway (18.52\%), and cellular response to lipopolysaccharide (22.22\%). Consistent with prior publications, HQ and BS are targeting different proteins in the nitric oxide biosynthetic process pathway.

\section{Pathway analysis to explore the underlying mechanisms of the HQ-BS herbal pair}

The KEGG pathway enrichment analysis suggested that the 54 putative targets of $\mathrm{HQ}$ and BS were enriched with FDR corrected $p<0.05$. As shown in Supplementary Table 3, the top 20 pathways associated with the HQ and BS herbal pair were obtained. Consistent with the previous publications, the tumor necrosis factor (TNF) signaling pathway, Tolllike receptor signaling pathway, and the IBD pathway had a strong correlation with the HQ-BS herbal pair against IBD. Furthermore, we investigated the distribution of partial targets of HQ-BS on the IBD pathway (shown in Fig. 7). After analysis by the Target-Pathway (T-P) network, 67 nodes (20 pathways and 47 proteins) and 303 interactions were obtained (shown in Fig. 8).

\section{The HQ-BS herbal pair attenuated $\mathrm{NO}$ and $\mathrm{PGE}_{2}$ release}

The results showed that an LPS insult induced a significant release of $\mathrm{NO}$ and $\mathrm{PGE}_{2}$ compared with the control cells. However, $\mathrm{HQ}, \mathrm{BS}$ and their active components treatment could significantly attenuate the levels of $\mathrm{NO}$ and $\mathrm{PGE}_{2}$ (as shown in Fig. 9). Especially, the crude materials of $\mathrm{HQ}$, BS and HQ-BS exerted much stronger activities in attenuating the release of $\mathrm{NO}$ and $\mathrm{PGE}_{2}$ compared with the Q-markers of HQ, BS and HQ-BS. These results indicate that the excellent anti-inflammatory effects of HQ-BS might be due to the synergistic effect of all their components.

\section{The HQ-BS herbal pair reduced the levels of iNOS and COX-2}

Western blot results showed that the levels of iNOS and COX-2 increased sharply after LPS stimulation

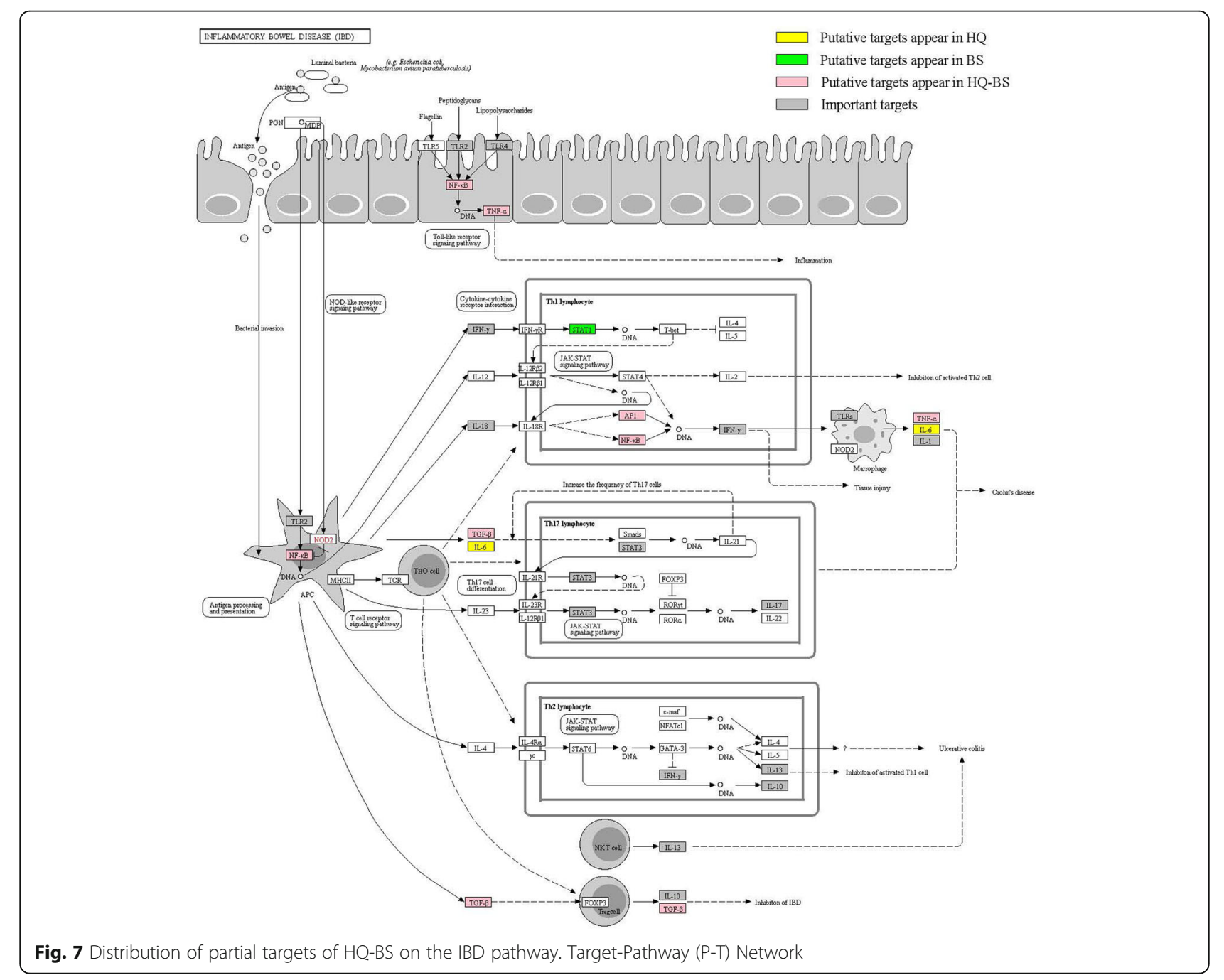




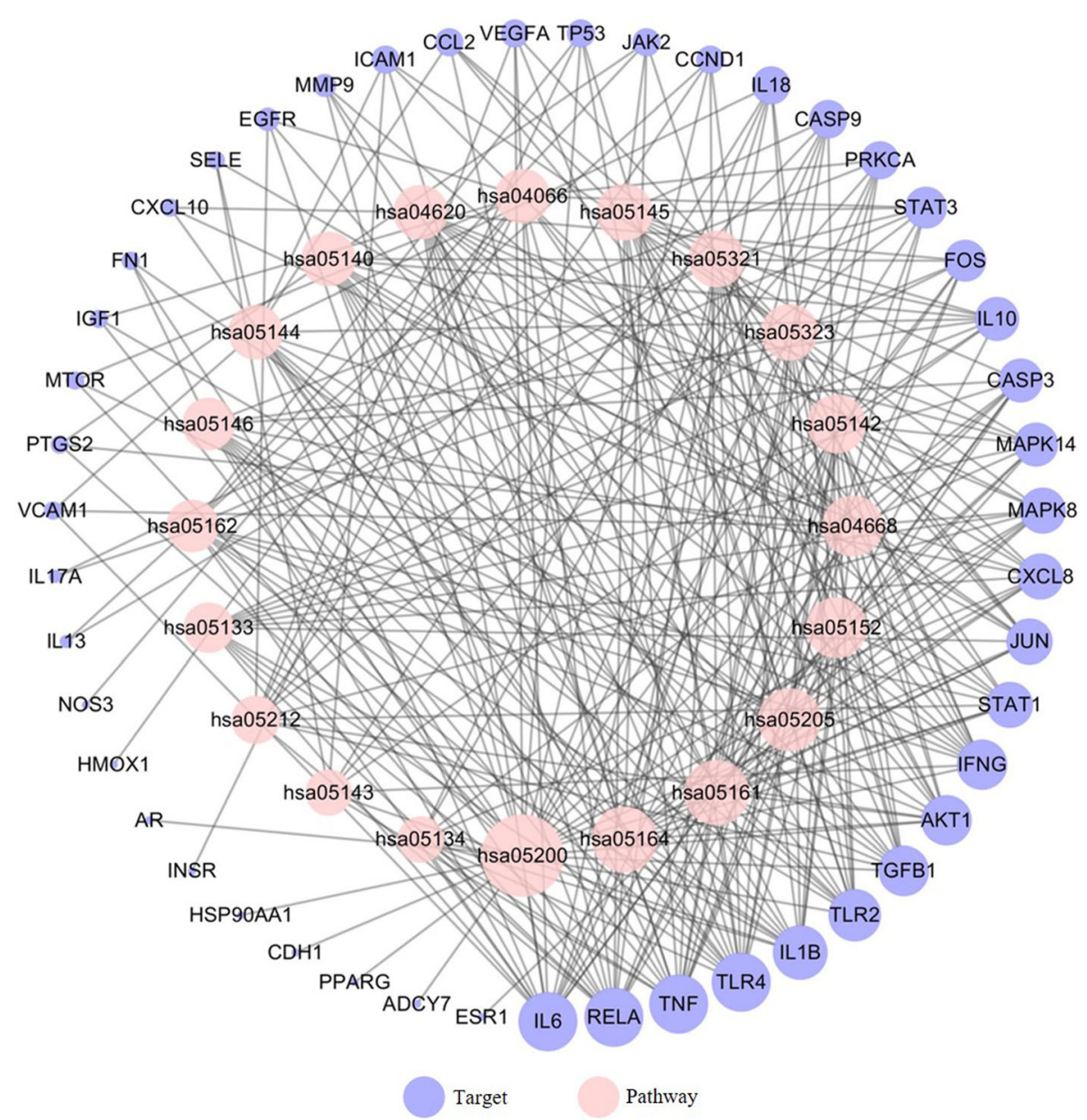

Fig. 8 Target-Pathway network for HQ-BS anti-IBD. The pink and blue nodes represent the pathway and targets, respectively, and the edges represent the interactions among them
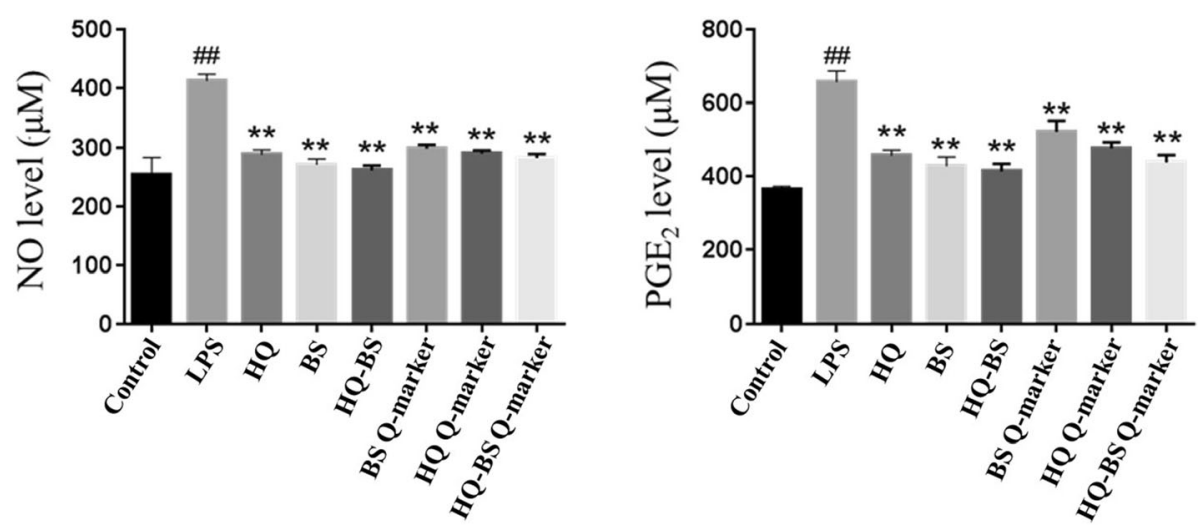

Fig. $9 \mathrm{HQ}, \mathrm{BS}, \mathrm{HQ}-\mathrm{BS}$ herbal pair and their Q-markers attenuated the levels of $\mathrm{NO}$ and $\mathrm{PGE} \mathrm{E}_{2}$. Data were expressed as the mean $\pm \mathrm{SD}(n=8)$. ${ }^{\# \#} p<$ 0.01 compared with control group; ${ }^{*} p<0.05,{ }^{* *} p<0.01$ compared with LPS group 


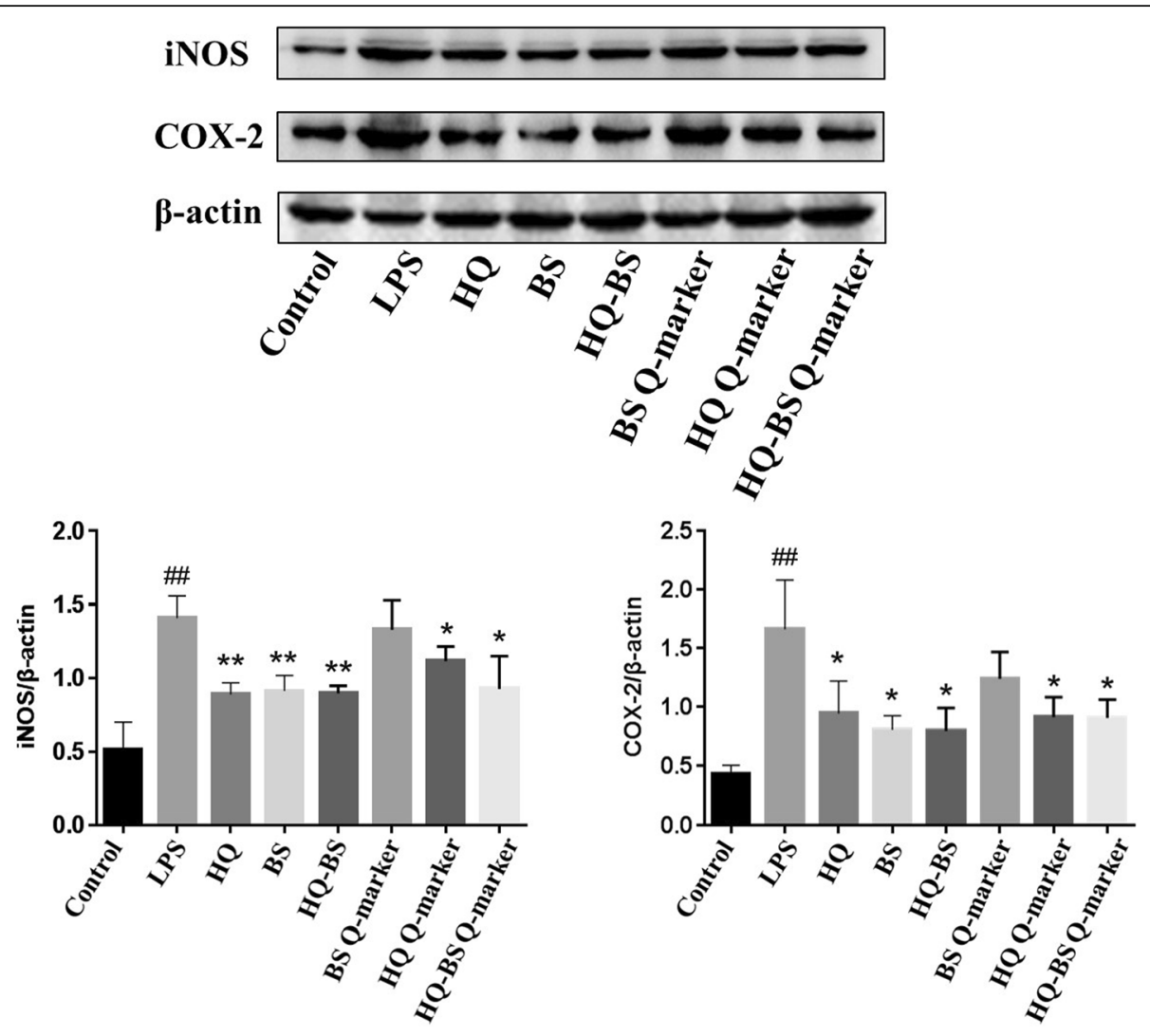

Fig. $10 \mathrm{HQ}, \mathrm{BS}, \mathrm{HQ}-\mathrm{BS}$ herbal pair and their Q-markers attenuated the levels of $\mathrm{NO}$ and $\mathrm{PGE}$. Data were expressed as the mean $\pm \mathrm{SD}(n=3)$ $\# p<0.01$ compared with control group; ${ }^{*} p<0.05,{ }^{* *} p<0.01$ compared with LPS group

compared with the control cells. However, HQ, BS and their active components treatment could significantly reduce the levels of $\mathrm{NO}$ and $\mathrm{PGE}_{2}$ (as shown in Fig. 10). Similar to the NO and $\mathrm{PGE}_{2}$ assay result, the crude materials of $\mathrm{HQ}, \mathrm{BS}$ and $\mathrm{HQ}-\mathrm{BS}$ could remarkably attenuate the inflammatory response remarkably than their Qmarkers. The Western blot results further confirmed that their synergistic effect plays an important role in the anti-inflammatory effects of HQ-BS.

\section{Discussion}

In the current study, we used a systems pharmacology approach to investigate the bioactive ingredients and significant pathways of the HQ-BS herbal pair. Then, we performed in vitro cell experiments to verify the network pharmacology prediction results.

A total of 215 compounds of the HQ and BS herbal pair were obtained from the TCMSP database, and 38 of them were chosen for further investigation following the drug-likeness analysis. Among the 38 compounds, 31 compounds were present in $\mathrm{HQ}, 5$ compounds were present in BS, and 2 compounds were present in both HQ and BS.
By analyzing these 38 bioactive compounds of $\mathrm{HQ}$ and $\mathrm{BS}$, we found that alkaloids and flavonoids were the primary active moieties of $\mathrm{HQ}$, and triterpenoids were the main active constituents of BS. In HQ, flavonoid constituents, such as baicalin, wogonin, acacetin, skullcapflavone, carthamin, dihydrooroxylin and oroxylin A are known to be anti-inflammatory agents [37]. Especially, baicalin was proven to exert excellent effects in ameliorating colitis through polarization of macrophages to an M2 phenotype, inflammation inhibition, and the suppression of oxidant stress and apoptosis [37-41]. The alkaloid composition, coptisine, was previously reported to attenuate intestinal damage [42, 43]. In BS, the triterpenoids composition, such as paeoniflorin, was reported to attenuate intestinal damage by inhibiting apoptosis and the NF-kappa B and TLR4 pathways $[15,16]$. By analyzing 54 putative anti-IBD targets of $\mathrm{HQ}$ and $\mathrm{BS}$, we found that the targets were mainly from the following two anti-IBD target classes: (1) apoptosis-related targets (such as heat shock proteins, apoptosis regulators, and caspases) and (2) 13 inflammation-related targets (such as prostaglandin, nitric oxide synthase, interleukin-6, and tumor necrosis factor) [32]. Prostaglandin plays a pivotal role in allergic inflammation. Moreover, reducing 
the level of prostaglandin exerts a protective effect against IBD and may represent a future safe treatment for IBD [44-46]. Furthermore, treatment with natural products could attenuate colitis by downregulating the level of prostaglandin. Nitric oxide synthase [47-49], interleukin-6 [50], and tumor necrosis factor [51] also revealed the same effect as prostaglandin in the regulation of IBD. Additionally, the apoptosisrelated targets, such as bax, bcl-2, caspase- 3 and caspase-9, also have crucial role in the pathogenesis of IBD [52]. By analyzing the top 20 KEGG pathways of the 54 putative targets, we found that positive regulation of the nitric oxide biosynthetic process was the most correlated pathway. The nitric oxide biosynthetic process plays an essential role in the pathobiology of IBD. In IBD, iNOS induces the generation of $\mathrm{NO}$, which leads to the release of pro-inflammatory cytokines, such as TNF- $\alpha$, interleukin-1 $\beta$ (IL-1 $\beta$ ) and interleukin-6 (IL-6). The accumulation of $\mathrm{NO}$ and pro-inflammatory cytokines in the intestinal epithelial cells triggers intestinal inflammation in IBD [53]. Cellular responses to lipopolysaccharide, inflammatory response and the TNF signaling pathway are also associated with the mechanism of HQ-BS against IBD.

The system pharmacology prediction results above suggest that cellular response to lipopolysaccharide, inflammatory response and the TNF signaling pathway are also associated with the mechanism of HQ-BS against IBD. Moreover, the HQ-BS herbal pair is predicted to target the nitric oxide biosynthetic process and inflammation-related targets (such as prostaglandin, nitric oxide synthase, interleukin-6, and tumor necrosis factor). Hence, to verify whether the HQ-BS herbal pair attenuates IBD symptom by targeting the inflammation response, we performed in vitro cell experiments to confirm their effects. The LPS-induced inflammation model in THP-1 cells is an accepted model to evaluate the actions of drugs on inflammation. A previous report demonstrated that paeoniflorin from BS is a Q-marker of HQD, while baicalin, baicalein, wogonin, and oroxylin A from BS are Q-markers of HQD [9]. Therefore, we tested the effect of HQ, BS, the HQ-BS herbal pair and their Q-markers against the LPS-induced inflammatory response in the THP-1 cells. The results showed that HQ, BS, the HQ-BS herbal pair and their Q-markers could suppress $\mathrm{NO}$ and $\mathrm{PGE}_{2}$ production, as well as iNOS and COX-2 protein expression. These cell-based results suggest that the synergistic effect plays an important role in the anti-inflammatory effects of HQ-BS.

Based upon the above prediction and experimental results, the underlying mechanism of the HQ-BS herbal pair against IBD may be associated with the regulation of the nitric oxide biosynthetic process and inflammatory cytokines release.

\section{Conclusion}

The mechanism of the HQ-BS herbal pair in IBD involves multiple ingredients, targets, and pathways. Combining the prediction and cell experimental results, the therapeutic effects of the HQ-BS herbal pair in IBD may be dependent on the regulation of the proteins and pathways related to the nitric oxide biosynthetic process and the inflammatory response by inhibiting NO synthesis and inflammatory cytokine release. The systems pharmacology approach combined with cell experimental methods as applied in our study provided an alternative strategy for the comprehensive understanding of the mechanisms of the HQ-BS herbal pair in IBD. In the future, we would like to validate the therapeutic effects of the HQ-BS herbal pair in IBD using a mouse model. Furthermore, we will investigate the mechanism whether by the HQ-BS herbal pair attenuates IBD through affecting the nitric oxide biosynthetic process and the inflammatory response.

\section{Supplementary information}

Supplementary information accompanies this paper at https://doi.org/10. 1186/s12906-020-03068-2.

Additional file 1: Table S1. Compounds of Scutellaria baicalensis (Huangqi) and Paeonia lactiflora (Baishao). Table S2. Targets of HQ-BS pair active compounds. Table S3. The top 20 KEGG pathways of 54 putative targets generated by DAVID.

Additional file 2. Original blot images.

\section{Abbreviations}

IBD: Inflammatory bowel disease; HQ: Huangqin; BS: Baishao; TCM: Traditional Chinese medicine; NO: Nitric oxide; $\mathrm{PGE}_{2}$ : Prostaglandin $\mathrm{E}_{2}$; iNOS: Inducible nitric oxide synthase; COX-2: Cyclooxygenase-2; LPS: Lipopolysaccharide; CD: Crohn's disease; UC: Ulcerative colitis; HQD: HuangQin decoction; GC: Gancao; DZ: Dazao; DSS: Dextran sodium sulfate; CDX2: Caudal-type homeobox 2; PXR: Pregnane X receptor; TNBS: Trinitro-benzene-sulfonic acid; TLR4: Toll like receptor-4; MyD88: Myeloid differential protein-88; NLRP3: NOD-like receptor 3; NF-kB: Nuclear factor kappa-B; MAPK: Mitogenactivated protein kinase; TCMSP: Traditional Chinese Medicine Systems Pharmacology Database; TTD: Therapeutic Targets Database;

CTD: Comparative Toxicogenomics Database; PPI: Protein-protein interaction; KEGG: Kyoto Encyclopedia of Genes and Genomes; H-C-T: Herb-CompoundTarget; GO: Gene Ontology; DAVID: Database for Annotation, Visualization and Integrated Discovery; FDR: False discovery rate; SD: Standard deviation; ANOVA: Analysis of variance; MW: Molecular weight; MLogP: Moriguchi octanol-water partition coeff. (logP); nHDon: Number of donor atoms for the $\mathrm{H}$-bonds; nHAcc: Number of acceptor atoms for $\mathrm{H}$-bonds; OB: Oral bioavailability; DL: Drug-likeness; ADME: Absorption, distribution, metabolism, and excretion; HIT: Herb Ingredients' Target; BP: Biological processes; TNF: Tumor necrosis factor; T-P: Target-Pathway; IL-1ß: Interleukin-1 $\beta$; IL6: Interleukin-6

\section{Acknowledgements}

Not applicable.

\section{Declarations}

Not applicable.

\section{Authors' contributions}

$\mathrm{XQH}$ designed this study, provided reagents and responsible for the system pharmacology research. ZWC participated in the system pharmacology research. MYL and YMZ performed the intro experiment. SJX and HYH 
analyzed that data. XLW drafted the manuscript. XBZ supervised the design of the study and reviewed this manuscript. All authors reviewed and approved the final version of this paper.

\section{Funding}

This study was supported by Special Project on the Integration of Industry, Education and Research of Guangdong Province (2014B090902002); Provincial-level Major Scientific Research Projects in Ordinary Universities of Guangdong Province, China (2017KZDXM017); Science and Technological Program for Dongguan's Social development, China (20185071501131614); Characteristic Cultivation Program for Subject Research of Guangzhou University of Chinese Medicine (XKP2019007); National Natural Science Foundation of China (81573932); National Natural Science Foundation of Guangdong, China (2019A1515010673); Guangzhou University of Chinese Medicine Discipline Research Major Project. All of these funding provided research resources for acquisition of materials and reagents.

\section{Availability of data and materials}

The dataset used during the current study is stored in a secured research data server at Guangzhou University of Chinese Medicine. The datasets used are available from the corresponding author upon reasonable request.

\section{Ethics approval and consent to participate}

Not applicable.

\section{Consent for publication}

All authors gave full consent for publication of the manuscript.

\section{Competing interests}

The author(s) declared no potential conflicts of interest with respect to the research, authorship, and/or publication of this article.

\section{Author details}

${ }^{1}$ Mathematical Engineering Academy of Chinese Medicine, Guangdong Provincial Key Laboratory of New Chinese Medicinal Development and Research, Guangzhou University of Chinese Medicine, 232\# Wai Huan East Road, Guangzhou Higher Education Mega Center, Guangzhou 510006, China. 2Dongguan Mathematical Engineering Academy of Chinese Medicine, Guangzhou University of Chinese Medicine, Dongguan 523808, China. ${ }^{3}$ School of Biomedical and Pharmaceutical Sciences, Guangdong University of Technology, 100\# Wai Huan West Road, Guangzhou Higher Education Mega Center, Guangzhou 510006, China. ${ }^{4}$ Dongguan Songshan Lake Yi Dao TCM Clinic, Dongguan 523808, China.

\section{Received: 5 October 2019 Accepted: 1 September 2020}

\section{Published online: 25 September 2020}

\section{References}

1. Ali T, Yun L, Rubin DT. Risk of post-operative complications associated with anti-TNF therapy in inflammatory bowel disease. World J Gastroentero. 2012;18(3):197-204.

2. de Chambrun GP, Blanc P, Peyrin-Biroulet L. Current evidence supporting mucosal healing and deep remission as important treatment goals for inflammatory bowel disease. Expert Rev Gastroent. 2016;10(8):915-27.

3. Jiang WY. Therapeutic wisdom in traditional Chinese medicine: a perspective from modern science. Trends Pharmacol Sci. 2005;26(11):55863.

4. Xiong XJ, Yang XC, Liu YM, Zhang Y, Wang PQ, Wang J. Chinese herbal formulas for treating hypertension in traditional Chinese medicine: perspective of modern science. Hypertens Res. 2013;36(7):570-9.

5. Zhang AH, Sun H, Qiu S, Wang XJ. Advancing Drug Discovery and Development from Active Constituents of Yinchenhao Tang, a Famous Traditional Chinese Medicine Formula. Evid-Based Compl Alt. 2013;2013: 257909

6. Wan P, Chen H, Guo Y, Bai AP. Advances in treatment of ulcerative colitis with herbs: from bench to bedside. World J Gastroentero. 2014;20(39): 14099-104.

7. Chen Q, Zhang H. Clinical study on 118 cases of ulcerative colitis treated by integration of traditional Chinese and Western medicine. J Tradit Chin Med. 1999;19(3):163-5.
8. Wang X, Cui DN, Dai XM, Wang J, Zhang W, Zhang ZJ, Xu FG. HuangQin decoction attenuates CPT-11-induced gastrointestinal toxicity by regulating bile acids metabolism homeostasis. Front Pharmacol. 2017;8.

9. Dai XM, Cui DN, Wang J, Zhang W, Zhang ZJ, Xu FG. Systems pharmacology based strategy for Q-markers discovery of HuangQin decoction to attenuate intestinal damage. Front Pharmacol. 2018;9:236.

10. Tilton R, Paiva AA, Guan JQ, Marathe R, Jiang Z, van Eyndhoven W, Bjoraker J, Prusoff Z, Wang H, Liu SH, et al. A comprehensive platform for quality control of botanical drugs (PhytomicsQC): a case study of Huangqin Tang (HQT) and PHY906. Chin Med. 2010;5:30.

11. Jiang WY, Seo GS, Kim YC, Sohn DH, Lee SH. PF2405, standardized fraction of Scutellaria baicalensis, ameliorates colitis in vitro and in vivo. Arch Pharm Res. 2015;38(6):1127-37.

12. Fajardo AM, Piazza GA. Chemoprevention in gastrointestinal physiology and disease. Anti-inflammatory approaches for colorectal cancer chemoprevention. Am J Physiol-Gastr L. 2015;309(2):G59-70.

13. Dou W, Mukherjee S, Li H, Venkatesh M, Wang HW, Kortagere S, Peleg A, Chilimuri SS, Wang ZT, Feng Y, et al. Alleviation of Gut Inflammation by Cdx2/ Pxr Pathway in a Mouse Model of Chemical Colitis. PLoS One. 2012;7:7.

14. Luo XP, Yu ZL, Deng C, Zhang JJ, Ren GY, Sun AN, Mani S, Wang ZT, Dou W. Baicalein ameliorates TNBS-induced colitis by suppressing TLR4/MyD88 signaling cascade and NLRP3 inflammasome activation in mice. Sci Rep-Uk. $2017 ; 7$.

15. Zhang JJ, Dou W, Zhang EY, Sun A, Ding LL, Wei XH, Chou GX, Mani S, Wang ZT. Paeoniflorin abrogates DSS-induced colitis via a TLR4-dependent pathway. Am J Physiol-Gastr L. 2014;306(1):G27-36

16. Gu PQ, Zhu L, Liu YJ, Zhang L, Liu JL, Shen H. Protective, effects of paeoniflorin on TNBS-induced ulcerative colitis through inhibiting NFkappaB pathway and apoptosis in mice. Int Immunopharmacol. 2017;50: 152-60.

17. Huang JH, Tang HT, Cao SM, He YC, Feng YB, Wang K, Zheng QS. Molecular Targets and Associated Potential Pathways of Danlu Capsules in Hyperplasia of Mammary Glands Based on Systems Pharmacology. Evid-Based Compl Alt. 2017:2017:1930598.

18. Yue SJ, Xin LT, Fan YC, Li SJ, Tang YP, Duan JA, Guan HS, Wang CY. Herb pair Danggui-Honghua: mechanisms underlying blood stasis syndrome by system pharmacology approach. Sci Rep-Uk. 2017;7:40318.

19. Zhong JL, Liu ZH, Zhou XX, Xu J. Synergic Anti-Pruritus Mechanisms of Action for the Radix Sophorae Flavescentis and Fructus Cnidii Herbal Pair. Molecules. 2017:22:9.

20. Pei $T L$, Zheng $C L$, Huang C, Chen XT, Guo ZH, Fu YX, Liu JL, Wang YH. Systematic understanding the mechanisms of vitiligo pathogenesis and its treatment by Qubaibabuqi formula. J Ethnopharmacol. 2016;190:272-87.

21. Liu JL, Pei TL, Mu JX, Zheng CL, Chen XT, Huang C, Fu YX, Liang ZS, Wang $\mathrm{YH}$. Systems Pharmacology Uncovers the Multiple Mechanisms of Xijiao Dihuang Decoction for the Treatment of Viral Hemorrhagic Fever. EvidBased Compl Alt. 2016:2016:9025036.

22. Wang C, Ren Q, Chen XT, Song ZQ, Ning ZC, Gan JH, Ma XL, Liang DR, Guan DG, Liu ZL, et al. System pharmacology-based strategy to decode the synergistic mechanism of Zhi-zhu Wan for functional dyspepsia. Front Pharmacol. 2018;9:841.

23. Wang Y, Guo G, Yang BR, Xin QQ, Liao QW, Lee SMY, Hu YJ, Chen KJ, Cong WH. Synergistic effects of Chuanxiong-Chishao herb-pair on promoting angiogenesis at network pharmacological and pharmacodynamic levels. Chin J Integr Med. 2017:23(9):654-62.

24. Ru JL, Li P, Wang JN, Zhou W, Li BH, Huang C, Li PD, Guo ZH, Tao WY, Yang YF, et al. TCMSP: a database of systems pharmacology for drug discovery from herbal medicines. J Cheminformatics. 2014;6.

25. Wishart DS, Feunang YD, Guo AC, Lo EJ, Marcu A, Grant JR, Sajed T, Johnson D, Li C, Sayeeda Z, et al. DrugBank 5.0: a major update to the DrugBank database for 2018. Nucleic Acids Res. 2018;46(D1):D1074-82.

26. Li YH, Yu CY, Li XX, Zhang P, Tang J, Yang QX, Fu TT, Zhang XY, Cui XJ, Tu $\mathrm{G}$, et al. Therapeutic target database update 2018: enriched resource for facilitating bench-to-clinic research of targeted therapeutics. Nucleic Acids Res. 2018;46(D1):D1121-7.

27. Davis AP, Grondin CJ, Johnson RJ, Sciaky D, McMorran R, Wiegers J, Wiegers TC, Mattingly CJ. The comparative Toxicogenomics database: update 2019. Nucleic Acids Res. 2019:47(D1):D948-54.

28. Whirl-Carrillo M, McDonagh EM, Hebert JM, Gong L, Sangkuhl K, Thorn CF, Altman RB, Klein TE. Pharmacogenomics knowledge for personalized medicine. Clin Pharmacol Ther. 2012;92(4):414-7. 
29. Szklarczyk D, Franceschini A, Wyder S, Forslund K, Heller D, Huerta-Cepas J, Simonovic M, Roth A, Santos A, Tsafou KP, et al. STRING v10: protein-protein interaction networks, integrated over the tree of life. Nucleic Acids Res. 2015:43(D1):D447-52.

30. Kanehisa M, Furumichi M, Tanabe M, Sato Y, Morishima K. KEGG: new perspectives on genomes, pathways, diseases and drugs. Nucleic Acids Res. 2017;45(D1):D353-61.

31. UniProt C. UniProt: a worldwide hub of protein knowledge. Nucleic Acids Res. 2019;47(D1):D506-15.

32. Shannon P, Markiel A, Ozier O, Baliga NS, Wang JT, Ramage D, Amin N, Schwikowski B, Ideker T. Cytoscape: a software environment for integrated models of biomolecular interaction networks. Genome Res. 2003;13(11): 2498-504.

33. Huang DW, Sherman BT, Lempicki RA. Systematic and integrative analysis of large gene lists using DAVID bioinformatics resources. Nat Protoc. 2009;4(1): 44-57.

34. Ye H, Ye L, Kang H, Zhang DF, Tao L, Tang KL, Liu XP, Zhu RX, Liu Q, Chen $Y Z$, et al. HIT: linking herbal active ingredients to targets. Nucleic Acids Res. 2011;39:D1055-9.

35. Yu H, Chen JX, Xu X, Li Y, Zhao HH, Fang YP, Li XX, Zhou W, Wang W, Wang $\mathrm{YH}$. A Systematic Prediction of Multiple Drug-Target Interactions from Chemical, Genomic, and Pharmacological Data. PLoS One. 2012;7:5.

36. Li S, Zhang ZQ, Wu LJ, Zhang XG, Li YD, Wang YY. Understanding ZHENG in traditional Chinese medicine in the context of neuro-endocrine-immune network. IET Syst Biol. 2007;1(1):51-60.

37. Zhu W, Jin ZS, Yu JB, Liang J, Yang QD, Li FJ, Shi XK, Zhu XD, Zhang XL. Baicalin ameliorates experimental inflammatory bowel disease through polarization of macrophages to an M2 phenotype. Int Immunopharmacol. 2016:35:119-26.

38. Feng JS, Guo CC, Zhu YZ, Pang LP, Yang Z, Zou Y, Zheng XB. Baicalin down regulates the expression of TLR4 and NFkB-p65 in colon tissue in mice with colitis induced by dextran sulfate sodium. Int J Clin Exp Med. 2014;7(11): 4063-72.

39. Zhang CL, Zhang S, He WX, Lu JL, Xu YJ, Yang JY, Liu D. Baicalin may alleviate inflammatory infiltration in dextran sodium sulfate-induced chronic ulcerative colitis via inhibiting IL-33 expression. Life Sci. 2017:186:125-32.

40. Yu FY, Huang SG, Zhang HY, Ye H, Chi HG, Zou Y, Lv RX, Zheng XB. Effects of baicalin in CD4+CD29+T cell subsets of ulcerative colitis patients. World Gastroentero. 2014;20(41):15299-309.

41. Yao J, Cao X, Zhang R, Li YX, Xu ZL, Zhang DG, Wang LS, Wang JY. Protective effect of Baicalin against experimental colitis via suppression of oxidant stress and apoptosis. Pharmacogn Mag. 2016;12(47):225-34.

42. Zhang ZH, Wu LQ, Deng AJ, Yu JQ, Li ZH, Zhang HJ, Wang WJ, Qin HL. New synthetic method of 8-oxocoptisine starting from natural quaternary coptisine as anti-ulcerative colitis agent. J Asian Nat Prod Res. 2014;16(8): $841-6$.

43. Xie M, Zhang HJ, Deng AJ, Wu LQ, Zhang ZH, Li ZH, Wang WJ, Qin HL. Synthesis and structure-activity relationships of N-Dihydrocoptisine-8ylidene aromatic amines and N-Dihydrocoptisine-8-ylidene aliphatic amides as Antiulcerative colitis agents targeting XBP1. J Nat Prod. 2016; 79(4):775-83.

44. Montrose DC, Nakanishi M, Murphy RC, Zarini S, McAleer JP, Vella AT, Rosenberg DW. The role of PGE (2) in intestinal inflammation and tumorigenesis. Prostag Oth Lipid M. 2015;116:26-36.

45. Arai Y, Matsuura T, Matsuura M, Fujiwarad M, Okayasu I, Ito S, Arihiro S. Prostaglandin E-major urinary metabolite as a biomarker for inflammation in ulcerative colitis: prostaglandins revisited. Digestion. 2016;93(1):32-9.

46. Arai $Y$, Arihiro S, Matsuura T, Kato T, Matsuoka M, Saruta M, Mitsunaga M, Matsuura M, Fujiwara M, Okayasu I, et al. Prostaglandin E-major urinary metabolite as a reliable surrogate marker for mucosal inflammation in ulcerative colitis. Inflamm Bowel Dis. 2014;20(7):1208-16.

47. Zhu L, Gu PQ, Shen H. Protective effects of berberine hydrochloride on DSSinduced ulcerative colitis in rats. Int Immunopharmacol. 2019;68:242-51.

48. de Oliveira GAL, de la Lastra CA, Rosillo MA, Martinez MLC, Sanchez-Hidalgo M, Medeiros JVR, Villegas I. Preventive effect of bergenin against the development of TNBS-induced acute colitis in rats is associated with inflammatory mediators inhibition and NLRP3/ASC inflammasome signaling pathways. Chem Biol Interact. 2019;297:25-33.

49. Sun BY, Yuan JY, Wang SY, Lin J, Zhang WJ, Shao JD, Wang RQ, Shi B, Hu HY. Qingchang suppository ameliorates colonic vascular permeability in dextran-sulfate-sodium-induced colitis. Front Pharmacol. 2018;9.
50. Yang Y, Guan JY, Shaikh AS, Liang YR, Sun LC, Wang M, Li DY, Qiu CH, Li XZ. Histone Acetyltransferase Mof affects the progression of DSS-induced colitis. Cell Physiol Biochem. 2018;47(5):2159-69.

51. Hunter T, Schroeder K, Sandoval D, Deodhar A. Persistence, discontinuation, and switching patterns of newly initiated TNF inhibitor therapy in Ankylosing spondylitis patients in the United States. Rheumatol Ther. 2019.

52. Vukelic I, Detel D, Pucar LB, Potocnjak I, Buljevic S, Domitrovic R. Chlorogenic acid ameliorates experimental colitis in mice by suppressing signaling pathways involved in inflammatory response and apoptosis. Food Chem Toxicol. 2018;121:140-50.

53. Kolios G, Valatas V, Ward SG. Nitric oxide in inflammatory bowel disease: a universal messenger in an unsolved puzzle. Immunology. 2004;113(4):427-37.

\section{Publisher's Note}

Springer Nature remains neutral with regard to jurisdictional claims in published maps and institutional affiliations.
Ready to submit your research? Choose BMC and benefit from:

- fast, convenient online submission

- thorough peer review by experienced researchers in your field

- rapid publication on acceptance

- support for research data, including large and complex data types

- gold Open Access which fosters wider collaboration and increased citations

- maximum visibility for your research: over $100 \mathrm{M}$ website views per year

At BMC, research is always in progress.

Learn more biomedcentral.com/submissions 